\title{
A New Method to Experimentally Investigate Local Pressure Loss of Oil-Water Two-Phase Flow through Pore Throats
}

\author{
Dongxu Liu, Na Huang, and Lei Liu* \\ State Key Laboratory of Multiphase Flow in Power Engineering, Xi' an Jiaotong University, 710049 Xi' an, China
}

\begin{abstract}
To investigate the resistance performance of pore throats in porous media, a new method was used to conduct experiments to indirectly measure the local pressure loss of single-phase water and oilwater two-phase flow through pore-throat structures. Four microchannels were designed and manufactured with MEMS technology. One of the four microchannels is a straight duct with no throat and each of the other three has one throat within the passage. By comparison of total pressure drops between the straight duct with no throat and the channel with a throat at the same flow rate, the local pressure loss over a porethroat structure can be determined. In this paper, the pore-throat structure is defined as a combination of a contraction, an expansion and a throat to stimulate the pore throat in porous media. Experimental results show that local pressure loss, nonlinear with the flow rate, grows up with the decrease of throat size and the increase of oil volume fraction. Local loss coefficient, characterizing the local resistance performance of pore-throat structure, diminishes with the increase of Reynolds number. Reynolds number (in throat part) is in the range of 100-1100. A new empirical correlation of local loss coefficient is proposed for single-phase water and oil-water two-phase flow through pore-throat structure.
\end{abstract}

\section{Introduction}

Pores in porous media, in which there usually exist pore throats, can be treated as microchannels [1-5] Pore-scale flow in porous media also can be treated as microchannel flow. Resistance performance of twophase flow through pore throats is thus an issue of significance in both microfluidics and percolation mechanics. This issue has received considerable attention in many applications including but not limited to enhanced oil recovery in petroleum engineering [6-9], fluid transport in fuel cells [10-12] and heat transfer in porous materials [13-15], etc.

A literature survey demonstrates that numbers of researches for pore-scale or microfluidic two-phase flow have been carried out. Up to now, however, few experimental studies on the resistance loss of fluid flow over pore throats have been reported, especially for oilwater two-phase flow. Pore throats were widely concerned in pore-throat network models to simulate relative permeability, capillary pressure and interfacial dynamics in porous media [10, 16-18]. A pore-throat network model consists of a huge number of pore throats. It is still a challenge for pore-throat network model to determine the flow resistance over a single pore throat based on experimental data. Due to the geometrical complexity of pore throats in porous media, the fractal studies for flow resistance in porous media were carried on the basis of porous models $[19,20]$. Those fractal results for flow resistance await being verified from the point of view of experiment.
Some experimental researches were associated with the effect of a pore throat on fluid flow, but not concerned about the resistance of over it. For example, a pore throat was used to investigate the critical condition for the release of trapped droplets [21]. And impacts of pore throats on flow characteristic of viscoelasticity fluids were probed [22].

A pore throat, as a unit of the pore-throat network, can be called the pore-throat structure. It usually can be treated as a combination of a contraction, an expansion and a throat [20]. Quite a few of investigations have been taken for the local resistance of contractions and expansions. These studies were concentrated on singlephase water and gas-water two-phase flow across abrupt or gentle contractions or expansions in microchannels. It is reported that the local loss coefficients measured for contractions and/or expansions are not consistent with theoretical predictions[23, 24] and modified correlations can predict local pressure loss well $[23,25,26]$. Chalfi and Ghiaasiaan [27] studied the local pressure losses of liquid flow and gas-liquid two-phase flow caused by abrupt expansion and contraction in microchannels. Guo, et al. [28] found that the contraction loss coefficient decreases with the increase of Reynolds number in laminar water flow. Kawahara, et al. [29] presented the growth of the pressure loss across sudden contraction grown with the increase of downstream superficial velocity. Studies mentioned above focused on the local pressure losses of contractions or expansions; even so, contractions and expansions are only parts of a whole

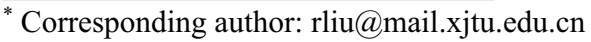


pore-throat structure but not a whole pore-throat structure itself.

Resistance loss of a pore-throat structure remains to be experimentally quantified for pore-scale oil-water flow. In this work, we are engaged in studying the local resistance loss of oil-water two-phase flow across porethroat structure, which is the basic component of porethroat networks in porous media. The influences of Reynolds number, pore-throat size and oil volume fraction on the resistance performance were discussed.

\section{Experiments}

\subsection{Experimental setup}

Experiments on flow in microchannels are carried out to study the local resistance performance of oil-water twophase flow across pore throats. As shown in Fig. 1, a high pressure nitrogen cylinder is used to provide power for flow of working fluid in microchannels. Data acquisition system is used to obtain the data of temperature and pressure automatically and instantaneously. Thermal resistors and transducers are used to measure flow temperatures. Differential pressure transmitters are used to measure the total pressure drop of fluid flow across microchannels. The data of volumetric flow rate are calculated with the mass flow rate measured by an electronic balance. Deionized water and mixture of white oil and deionized water are used as the working fluids. Oil volume fractions of oil-water two-phase emulsion are about $10 \%, 20 \%$ and $30 \%$ respectively, aiming at investigating the effects of twophase fluid on local pressure loss in the pore-throat structure. Physical parameters of deionized water and white oil are shown in Table 1.

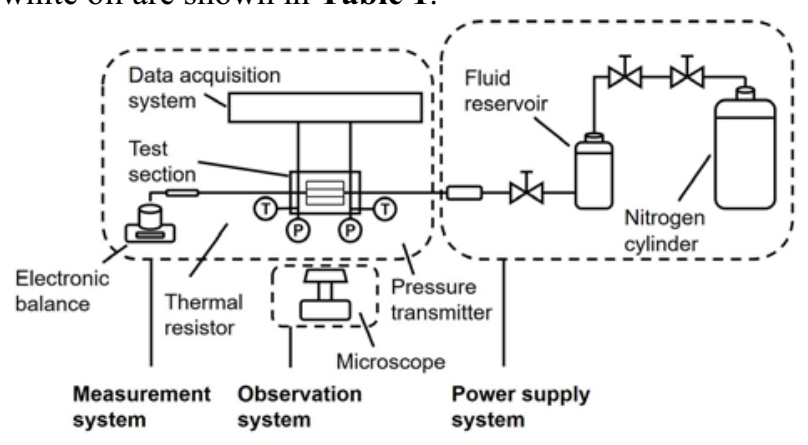

Fig. 1. Experimental system

Table 1. Physical parameters of working fluids

\begin{tabular}{lll}
\hline Fluids & $\rho\left(\mathrm{kg} / \mathrm{m}^{3}\right)$ & $\mu(\mathrm{mPa} \cdot \mathrm{s})$ \\
\hline Deionized water & 998.2 & 0.987 \\
White oil & 833.2 & 9.761 \\
\hline
\end{tabular}

Fig. 2 shows the test section with four rectangular cross section microchannels, denoted as channels 1, 2, 3 and 4. Pore-throat structures are designed in microchannels to simulate pore throats in porous media. It's clear that there is one throat within the passages of channels 1, 2 and 3, respectively. Meanwhile, channel 4 has a straight duct with no throat. Hydraulic diameters of such three throats are $37 \mu \mathrm{m}, 45 \mu \mathrm{m}$ and $57 \mu \mathrm{m}$, respectively. Fig. 3 gives the picture of a pore-throat structure consisting of three parts - a contraction, a throat and an expansion. The ducts of these four channels, except for the micro throats, have the same geometry with the hydraulic diameter of $181 \mu \mathrm{m}$ and the length of $30 \mathrm{~mm}$. These four ducts were manufactured on a silicon slice by MEMS technique and covered with a piece of Pyrex glass. Table 2 shows the geometric parameters of the microchannels.

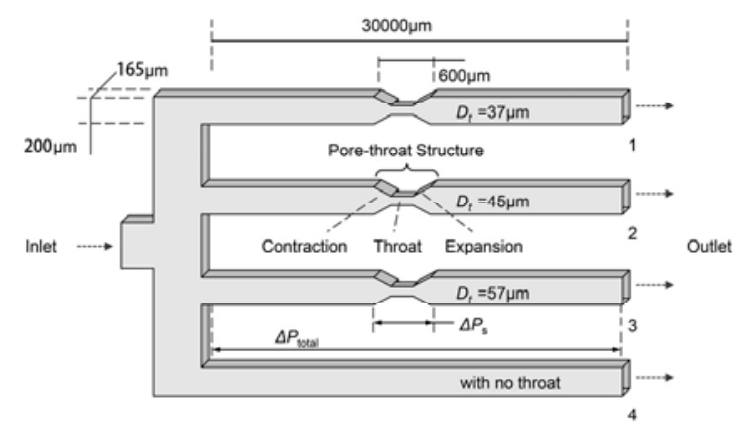

Fig. 2. Test section with four rectangular microchannels

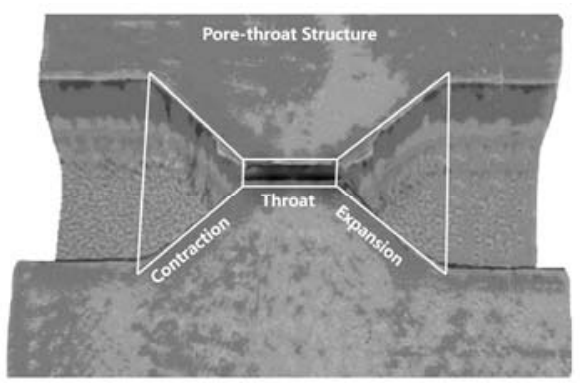

Fig. 3. Picture of a pore-throat structure taken by a digital microscope

Table 2. Geometry of the microchannels

\begin{tabular}{llllll}
\hline No. & $W_{\mathrm{m}}(\mu \mathrm{m})$ & $H_{\mathrm{m}}(\mu \mathrm{m})$ & $W_{\mathrm{t}}(\mu \mathrm{m})$ & $H_{\mathrm{t}}(\mu \mathrm{m})$ & $L_{\mathrm{t}}(\mu \mathrm{m})$ \\
\hline 1 & 200.0 & 165.1 & 89.8 & 23.6 & 100.9 \\
2 & 200.0 & 165.1 & 66.1 & 34.5 & 99.4 \\
3 & 200.0 & 165.1 & 75.3 & 45.4 & 101.5 \\
4 & 200.0 & 165.1 & --- & --- & --- \\
\hline
\end{tabular}

\subsection{Experimental procedure}

Obviously, it's hard to deploy sensors inside the passage of these microchannels, let alone in throats, to measure the pressure distribution along the flow path. So a new method was introduced to indirectly confirm the experimental value of local pressure loss through pore throats. Theoretically, total pressure drop in each of these microchannels with one pore-throat structure consists of two parts: frictional pressure drop along the microchannel excluding the pore-throat structure within it and local pressure loss of the pore-throat structure, which can be expressed as

$$
\Delta P_{\text {total }}=\Delta P_{\mathrm{f}}+\Delta P_{\mathrm{s}}
$$

where, $\Delta P_{\text {total }}$ is the total pressure drop, $\Delta P_{\mathrm{f}}$ is the frictional pressure drop, and $\Delta P_{\mathrm{s}}$ is the local pressure loss of pore-throat structures. For channel 4 , there is no local 
pressure loss of pore-throat structure and the total pressure drop is the frictional pressure drop along the total passage. As mentioned above, channel 4 has the same geometric parameters as channels 1,2 and 3 except for the pore-throat structure. So, local pressure loss, characterizing the resistance performance, of flow across pore-throat structures can be measured experimentally by comparing pressure drop of the channel with one throat and that of the straight duct with no throat at the same volume flow rate. Local pressure loss of porethroat structures in channels 1, 2 and 3 shown in Fig. 2 can be derived from Equation

(1). As these four channels have the same geometry excluding pore-throat structures in channels 1,2 and 3 and the length of these three pore-throat structures is about $300 \mu \mathrm{m}, 1 \%$ of the total length, total pressure drop in channel 4 can approximately refer to the frictional pressure drop channels 1,2 and 3 . So experimental procedure in this work becomes as simple as getting the data of pressure drop and volumetric flow rate at nearly constant temperature for each run, where various driving pressures are set.

\subsection{Theoretical analyses}

As mentioned, the working fluids in this work are singlephase deionized water and emulsions of white oil in deionized water. Most oil droplet sizes in emulsions are about $10 \mu \mathrm{m}$, much smaller than the hydraulic diameter as well as the narrowest cross-section side of throats, shown in Fig. 4, presenting flow across a pore-throat structure for emulsion with $30 \%$ oil volume fraction. So a homogeneous model is used, where no capillary pressure caused by oil droplets was considered. Fig. 5 shows a diagram of fluid flow across a pore-throat structure.

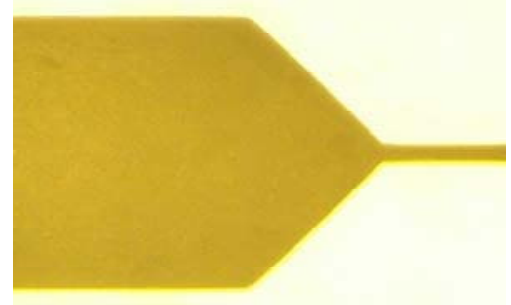

Fig. 4. Emulsion flow across a pore-throat structure

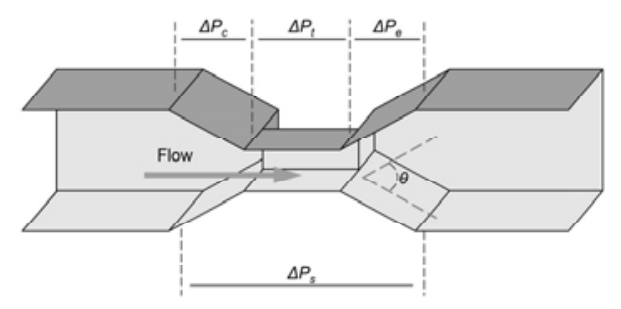

Fig. 5. Diagram of flow passing through a pore-throat strucure

Local pressure drop of pore-throat structures consists of three parts, pressure drops of the contraction, the throat and the expansion. It is given as

$$
\Delta P_{\mathrm{s}}=\Delta P_{\mathrm{c}}+\Delta P_{\mathrm{t}}+\Delta P_{\mathrm{e}}
$$

where $\Delta P_{\mathrm{s}}$ is the local pressure drop of pore-throat structure, $\Delta P_{\mathrm{c}}$ and $\Delta P_{\mathrm{e}}$ are pressure drops passing through contraction and expansion, respectively, and $\Delta P_{\mathrm{s}}$ is the pressure drop passing through throat, a frictional pressure drop in deed. Strictly, local pressure loss is only the irreversible part of local pressure drop. However, the local pressure drop of pore-throat structure is totally irreversible. In other words, it also can be called local pressure loss, while local pressure drops of contraction, expansion and throat are not pressure losses. By using the general formula of local resistance loss for flow area changes [30], local pressure loss of pore-throat structure can be written as

$$
\Delta P_{\mathrm{s}}=\Delta P_{\text {loss } \mathrm{s}}=K_{\mathrm{s}} \frac{1}{2} \rho u_{\mathrm{t}}^{2}
$$

where, $K_{\mathrm{s}}$ is the local resistance coefficient of pore-throat structure, $\rho$ is the density of the working fluid and $u_{\mathrm{t}}$ is the superficial velocity passing though throat. Generally, the local pressure loss is a kind of energy loss and it can be written as a product of the kinetic energy and a coefficient, i.e. local resistance coefficient.

$R e_{\mathrm{t}}$ is the Reynolds number in throat, defined as

$$
\operatorname{Re}=\frac{\rho u D}{\mu}
$$

where $\mu$ is dynamic viscosity of working fluid, D is the characteristic dimension.

Note that physical parameters for oil-water twophase flow are determined by the homogeneous model. Density and viscosity, $\rho_{\mathrm{m}}$ and $\mu_{\mathrm{m}}$, of the emulsions are calculated by

$$
\begin{aligned}
& \rho_{\mathrm{m}}=(1-x) \rho_{\mathrm{w}}+x \rho_{\mathrm{o}} \\
& \mu_{\mathrm{m}}=\left(1-\phi_{\mathrm{o}}\right) \mu_{\mathrm{w}}+\phi_{\mathrm{o}} \mu_{\mathrm{o}}
\end{aligned}
$$

where $\mathrm{x}$ is the mass fraction of oil in oil-water emulsion, given as

$$
x=\frac{\phi_{\mathrm{o}} \rho_{\mathrm{o}}}{\left(1-\phi_{\mathrm{o}}\right) \rho_{\mathrm{w}}+\phi_{\mathrm{o}} \rho_{\mathrm{o}}}
$$

\section{Results and discussion}

For flow in ducts, pressure drop and flow rate are two fundamental parameters and the relationship between them is the basis of analyses and discussions. Fig. 6 (a) and 6 (b) depict the relationship between total pressure drop and volumetric flow rate for single-phase water flow and the oil-water two-phase flow with oil volume fraction of $10 \%$, respectively, in four different microchannels, showing clearly that the total pressure drop in channel 4 increases linearly along with the volumetric flow rate both for single-phase water flow and $10 \%$ oil volume fraction oil-water two-phase flow. Total pressure drops in channels 1,2 and 3 are much larger that in channel 4 and increase nonlinearly with volumetric flow rate, which are shown apparently in Fig. 6 (a) for water flow and Fig. 6 (b) for 10\% oil-in-water two-phase flow. It indicates that the exists of pore-throat structures can significantly raise the total pressure drop in a microchannel for both cases of single-phase flow and two-phase flow, especially when volumetric flow 
rate is large, as a result, inducing nonlinear relationship between total pressure drop and volume flow rate in these three microchannels.

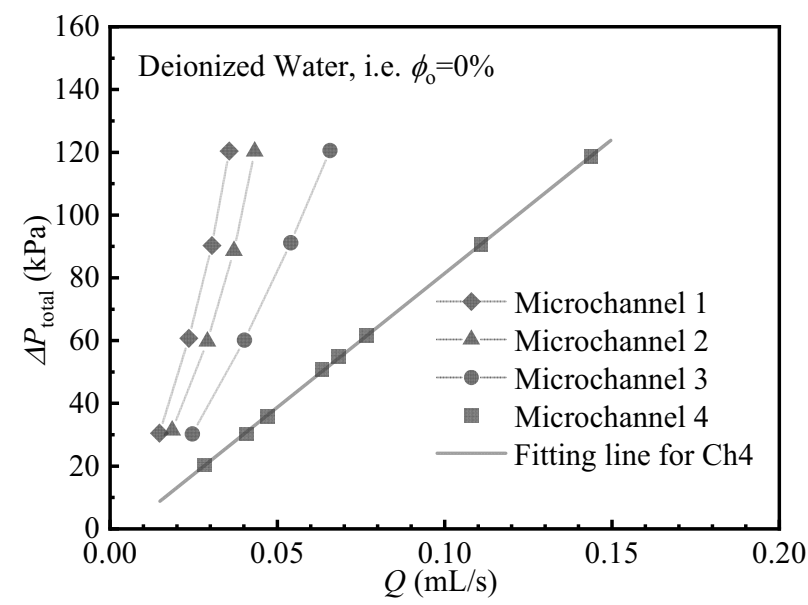

(a)

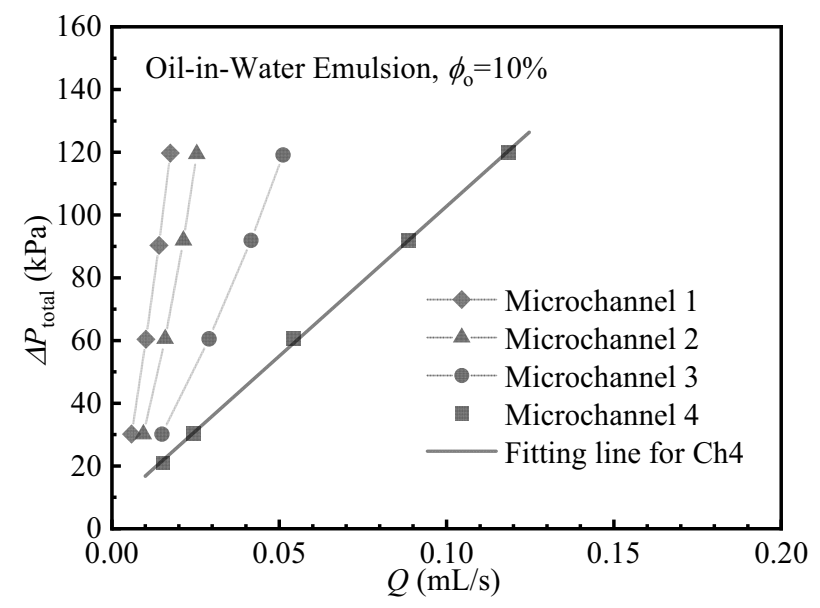

(b)

Fig. 6. Total pressure drop versus volumetric flow rate : (a) single-phase water flow; (b) oil-water two-phase flow, $=10 \%$

The characteristic dimension of these four microchannels mentioned above is so small that there is no sensor available to be fixed in the passage to directly obtain the data of pressure profile along the duct, let alone the measurement of local pressure loss in the porethroat structure. And even though probes can be fixed or pressure taps can be located on the inner wall of passages, the flow field could be destroyed. In order to obtain the data of local pressure loss in pore-throat structure, a particular method was adopted. A linear function was obtained by linearly fitting the total pressure drops measured versus the volumetric flow rates for channel 4 . The ratio between the length of the pore-throat structure and the total length of the microchannel is less than $1 \%$ for all of channels 1,2 and 3. Additionally, total pressure drop is proportional to the channel length for provided volumetric flow rate according to the linearity of total pressure drop versus volumetric flow rate in channel 4 . The depth and width of the four microchannels are all the same except for the pore-throat structures. This means the local pressure loss of flow passing through pore-throat structure in any of channels 1, 2 and 3 can be considered as the difference between the total pressure drop of such channels and the total pressure drop of channel 4 at a same volumetric flow rate. Likewise, the experimental data of local pressure loss of pore-throat structures in channel 3 for $20 \%$ and $30 \%$ oil volume fraction oil-water two-phase flows are obtained.

Fig. 7 (a) shows the local pressure loss of flow passing through pore-throat structures calculated by methods mentioned above versus superficial velocity in throats. The experimental data and their fitting curves are presented for deionized water flow and oil-water two-phase flow (oil volume fraction, 10\%). Additionally, Fig. 7 (b) shows the experimental data as well as their fitting curves for water and oil-in-water two-phase flow in various cases of oil volume fraction in channel 3. It can be seen from the experimental data in Fig. 7 (a) that the local pressure loss of flow passing through porethroat structure increases nonlinearly with the superficial velocity in throat part. For a particular working fluid, either single-phase water or $10 \%$ oil-in-water two-phase emulsion, local pressure loss across pore-throat structure grows up with the decrease of hydraulic diameter of the throat part. Additionally, for a particular pore-throat structure, one of those in channel 1, 2 or 3, local loss for $10 \%$ oil-water two-phase flow is larger than that for deionized water. This is much more clear in Fig. 7 (b) that local pressure loss grows up with the increase of oil volume fraction.
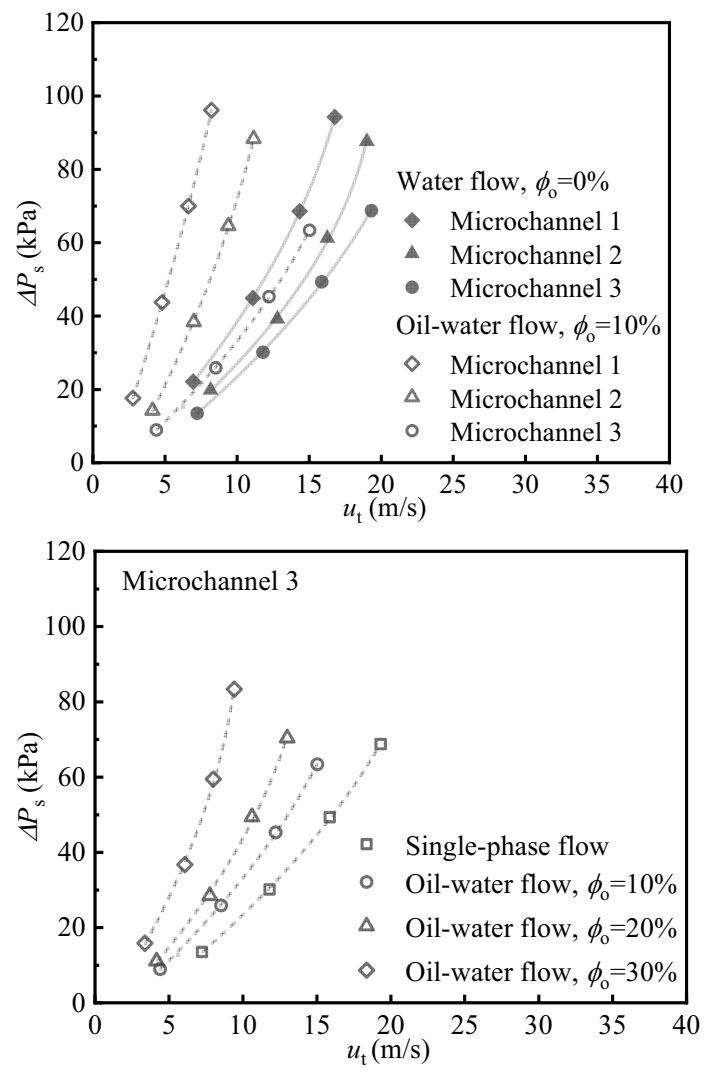

Fig. 7. Local pressure loss versus surperficial velocity in throat (a) single-phase water adn oil-water two-phase flow; (b) oilwater two-phase flow in the pore-throat structure of channel 3 with various oil volume fraction 
It indicates that both throat size and physical parameters of working fluid, such as viscosity, are main factors influencing the resistance performance of flow across pore-throat structures. Generally, oil-in-water two-phase flow may give rise to much larger local loss than single-phase water flow, implying that the viscous friction is the main cause of the local resistance, for viscosity of working fluid goes up with the increase of oil volume fraction. However, effects of throat size on local pressure loss cannot be ignored. One the one hand, it's clear that there is a negative correlation between local pressure loss and throat size. On the other hand, local loss of single-phase water flow may be larger than that of oil-water two-phase flow because of the throat size, curve of the experimental data for water flow through pore throat in channel 1 , for instance, locating above that for $10 \%$ oil-water two-phase flow.

It can be concluded that local resistance of pore throats is dominated by fluid viscosity and characteristic dimension of throats. So it is necessary to investigate the relationship between local resistance performance and Reynolds number. Fig. 8 displays the local loss coefficient of pore-throat structure versus Reynolds number in throat part. The Reynolds number is ranging from 100 to 1100 , indicating flow in pore-throat structure is laminar flow. From an overall perspective, local loss coefficient goes down with the increase of Reynolds number in throat part. Generally, smaller oil volume fraction, up to single-phase water, and larger hydraulic diameter of throats lead to larger Reynolds number, therefore smaller local loss coefficient. For a particular working fluid in a particular pore-throat structure, local loss coefficient varies sharply among different runs when Reynolds number is small and varies gently when Reynolds number is large. Characteristic dimension is an important factor influencing the local resistance performance, shown in Figure 8, local loss coefficient varying dramatically with the hydraulic diameter of throats, especially for oil-water two-phase flow. Similarly, effects of oil volume fraction on local pressure loss coefficient are prominent. In channel 3, for example, local loss coefficient as well as Reynolds number grows up with the increase of oil volume fraction. Moreover, changes of local loss coefficient caused by throat hydraulic diameter are much more abrupt for $10 \%$ oil volume fraction oil-water two-phase flow than that for single-phase water flow.

All experimental data considered as a whole, an empirical correlation is obtained:

$$
K_{\mathrm{s}}=210.8 R e_{t}^{-0.92}
$$

Intuitively, however, deviations of some experimental data are considerable. On the one hand, it cannot be denied that the new correlation can demonstrate the trend of local loss coefficient of porethroat structures versus Reynolds number in throats to some degree. On the other hand, flow for different volume fraction in different pore-throat structures deviates from predictions by the new correlation in the relationship of local pressure loss coefficient versus Reynolds number in throats.

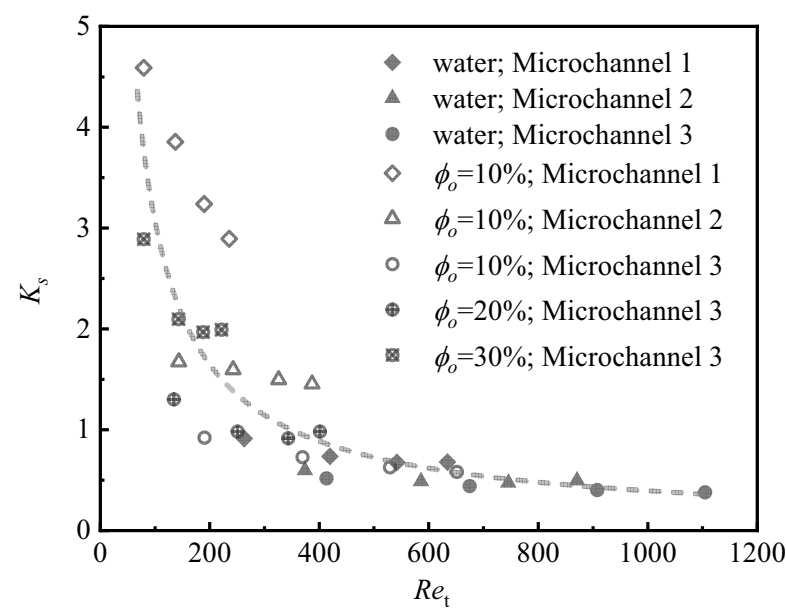

Fig. 8. Local pressure loss coefficient of pore-throat structure versus Reynolds number in throat part

\section{Conclusion}

Experiments on single-phase water and oil-water twophase flow through pore-throat structures were carried out to investigate the local resistance performance of a single pore throat in porous media.

Traditionally, it's not easy to directly measure the pressure distribution along the passages of microchannels, especially with a throat in it. So a new method was used to indirectly obtain the experimental data of local pressure loss of flow passing through each pore-throat structure by comparing the difference between the total pressure drop of a microchannel with a pore-throat structure and that of a straight microchannel with no throat, where these two microchannels are exactly the same except the pore-throat structure. The total pressure drop of the straight microchannel with no pore-throat structure increased linearly and that of the microchannel with a pore-throat structure increased linearly with the volumetric flow rate and the latter total pressure drop was much larger than the former one, indicating that the nonlinearity and deviation was caused by the exist of the pore-throat structure.

Local pressure loss of pore-throat structure was dominated by the viscosity of working fluids and the character dimension of pore-throat structures. The relationship of local pressure loss coefficient of porethroat structures versus Reynolds number in throat part was used to characterize the local resistance performance, showing that the local loss coefficient decreased with the increase of Reynolds number. A new empirical correlation of local loss coefficient was proposed as a unary function of Reynolds number, showing a good agreement in trend with the experimental data.

\section{Acknowledgments}

This work is supported by the National Natural Science Foundation of China (No.11572241). 


\section{References}

1. M.J. Blunt, M.D. Jackson, M. Piri, P.H. Valvatne, Adv. Water Resour. 25 (8-12), 1069 (2002).

2. P.H. Nelson, Aapg Bulletin 93 (3), 329 (2009).

3. C.R. Clarkson, N. Solano, R.M. Bustin, A.M.M. Bustin, G.R.L. Chalmers, L. He, Y.B. Melnichenko, A.P. Radlinski, T.P. Blach, Fuel 103, 606 (2013).

4. P. Li, M. Zheng, H. Bi, S. Wu, X. Wang, J. Petrol. Sci. Eng. 149, 665 (2017).

5. J. Lai, G. Wang, Z. Wang, J. Chen, X. Pang, S. Wang, Z. Zhou, Z. He, Z. Qin, X. Fan, Earth-Sci. Rev. 177, 436 (2018).

6. R.T. Armstrong, D. Wildenschild, J. Petrol. Sci. Eng. 94-95, 155 (2012).

7. S.-Y. Hsu, M. Hilpert, Adv. Water Resour. 95, 235 (2016).

8. Y. Zhou, D. Yin, R. Cao, C. Zhang, J. Petrol. Sci. Eng. 163, 519 (2018).

9. W. Cao, K. Xie, X. Lu, Y. Liu, Y. Zhang, Fuel 237, 1151 (2019).

10. A. Bazylak, V. Berejnov, B. Markicevic, D. Sinton, N. Djilali, Electrochim. Acta 53 (26), 7630 (2008).

11. V. Berejnov, A. Bazylak, D. Sinton, N. Djilali, J. Electrochem. Soc. 157 (5) (2010).

12. L. Li, S.Y. Bei, Q. Xu, K.Q. Zheng, Y. Zheng, Int. J. Energ. Res. 42 (3), 1277 (2018).

13. G. Xia, L. Chai, M. Zhou, H. Wang, Int. J. Therm. Sci. 50 (3), 411 (2011).

14. J. Xu, X. Ji, W. Yang, Z. Zhao, Int. J. Heat Mass Tran. 72, 163 (2014).

15. K. Milani Shirvan, R. Ellahi, S. Mirzakhanlari, M. Mamourian, Appl. Therm. Eng. 109, 761 (2016).

16. G.R. Jerauld, S.J. Salter, Transport Porous Med. 5 (2), 103 (1990).

17. R.G. Hughes, M.J. Blunt, Adv. Water Resour. 24 (3), 409 (2001).

18. F. Moebius, D. Or, Phys. Rev. E Stat. Nonlin. Soft Matter Phys. 90 (2), 023019 (2014).

19. J. Wu, B. Yu, Int. J. Heat Mass Tran. 50 (19-20), 3925 (2007).

20. K.C. Zheng, T. Wang, H.C. Luo, Z.J. Gong, W.F. Wu, Int. J. Heat Mass Tran. 115, 225 (2017).

21. L. He, Z. Luo, B. Bai, J. Colloid Interf. Sci. 554, 1 (2019).

22. Y. Li, R. Wang, R. Wang, W. Liu, C. Dai, X. Yao, M. Zhao, Y. Sun, G. Zhao, Y. Wu, J. Petrol. Sci. Eng. 174, 821 (2019).

23. Z. Li, J. Yu, C.F. Ma, Sci. China Ser. E 51 (2), 162 (2008).

24. S. Khodaparast, N. Borhani, J.R. Thome, Microfluid. Nanafluid. 17 (3), 561 (2014).

25. I.Y. Chen, C.-Y. Tseng, Y.-T. Lin, C.-C. Wang, Int. J. Multiphase Flow 35 (3), 297 (2009).
26. C.C. Wang, C.Y. Tseng, I.Y. Chen, Int. J. Heat Mass Tran. 53 (19-20), 4287 (2010).

27. T.Y. Chalfi, S.M. Ghiaasiaan, Int. J. Multiphase Flow 34 (1), 2 (2008).

28. H. Guo, L. Wang, J. Yu, F. Ye, C. Ma, Z. Li, Front. Engerg. Power Eng. China 4 (2), 149 (2010).

29. A. Kawahara, M.H. Mansour, M. Sadatomi, W.Z. Law, H. Kurihara, H. Kusumaningsih, Exp. Therm. Fluid Sci. 66, 243 (2015).

30. F.M. White, Fluid Mechanics, 8th ed. (McGraw-Hill, New York, 2015). 